\title{
The Application of CLEA Model in Accessing Health Risks of PAHs in Soil
}

\author{
Sihong Chao, Yanxue Jiang, Li Qiao, Hongbin Cao*
}

College of Resource Science and Technology, Beijing Normal University, Beijing 100875, China

\section{CLEA 模型在土壤多环芳烃健康风险评价中的应用 \\ 昆思宏, 蒋艳雪, 乔莉, 曹红斌 \\ ${ }^{1}$ 北京师范大学资源学院, 北京 100875 , 中国}

\begin{abstract}
The concentrations of PAHs in 29 soil samples in a little town in southern Jiangsu were detected. Based on these soil concentration data and parameters from existing studies for this area, we use contaminated land exposure assessment (CLEA) model developed by Environment Agency of United Kingdom to analyze the Average Daily Exposure (ADE) and subsequent health risks of the local residents for their exposure to PAHs in soil. The health risk assessment shows that oral of and dermal contact to soil are the two main pathways for PAHs exposure. Compared to children and teenagers (0-16), the adults (16-65) have higher accumulative carcinogenic risk. However, the annual mean carcinogenic risk of the children is higher than adults. Generally speaking, women have a little higher carcinogenic risk than men. The health risks of all the groups exceed $10^{-6}$, which need to be concerned.
\end{abstract}

Keywords: CLEA model, PAHs, Health risk assessment

\section{摘要}

本文以苏南某镇 29 个土壤样品中 16 种多环芳烃 (PAHs) 的浓度数据为基础, 采用英国环境保护署 开发的污染场地暴露评估模型 (CLEA), 通过模型 参数本地化, 计算了当地居民对土壤中多环芳烃的暴 露量, 并进一步评价相应的健康风险。结果表明: 经 口摄入和皮肤接触是健康风险较大的两种暴露途径,

*通讯作者: 201421190003@mail.bnu.edu.cn
成人(16-65)与少年儿童(0-16)相比, 累积致癌风险较 大, 但儿童的年平均致癌风险高于成人, 女性的致癌 风险高于男性。所有人群的致癌风险都超过了 $10^{-6}$, 应当引起关注。

关键词: CLEA 模型, 多环芳烃, 健康风险评价

1. 引言

多环芳烃 (PAHs) 是指由两个或两个以上苯环以稠 环形式相连组成的一类有机污染物, 广泛存在于环境 介质中。PAHs主要来源于化石燃料、生物质等的不 完全燃烧以及石油及其衍生品的挥发、泄漏等 ${ }^{[1]}$ 。其 中, 由于16种多环芳烃包括菜( NAP)、二氢范 (ACE)、 范烯（ACY）、苆（FLO）、菲（PHE）葱（ANT）、 苂葱 (FLA)、萠 (PYR)、苯并 $[a]$ 葱 $(B a A) 、$ 屈 $(C H R) 、$ 苯并 $[b]$ 苂葱 $(B b F)$ 、苯并 $[k]$ 苂葱 $(B k F)$ 、苯并 $[a]$ 芘 $(\mathrm{BaP}) 、$ 二苯并 $[\mathrm{a}, \mathrm{h}]$ 萝 $(\mathrm{DahA}) 、$ 狮并 $[1,2,3$-cd $]$ 萠 (IcdP) 和苯并 [ghi] 菲 (BghiP), 具有持久性、 毒性和生物蓄积性, 美国环境保护署 (USEPA) 将其 列为优先控制污染物 ${ }^{[2]}$ 。多环芳烃的健康风险评价 通常是基于毒性当量法将 16 种多环芳烃的浓度等效 为致癌的苯并 $[\mathrm{a}]$ 芘 $(\mathrm{BaP})$ 的浓度并结合人体暴露途 径计算得到其暴露量, 最后根据 $\mathrm{BaP}$ 的致癌斜率因子 (SF) 计算得到其终生致癌风险（ILCR）。

土壤中多环芳烃对人体造成健康危害的主要途 径 ${ }^{[3,4]}$ 包括经口摄入 (意外) 、皮肤接触和吸入 (呼吸 含有污染土壤的空气)。其中, 经口摄入是最主要的 途径 ${ }^{[4]}$ 。但对于种植农作物的居民来说, 通过膳食摄 入污染土壤生产出的农作物对其健康的影响也不容 忽视。CLEA (Contaminated Land Exposure Assessment) 模型 ${ }^{[5]}$ 是英国环境保护署推荐用来进行污染场地评 价以获取土壤指导限值 (Soil Guideline Values, SGVs) 
的模型, 暴露场景包括近地表人类活动和食用污染场 地种植的农作物 ${ }^{[6]}$ 。虽然该模型自身并不计算污染 物的健康风险, 但由于其对目标人群在指定暴露场景 下不同暴露途径的平均日暴露量 (Average Daily Exposure, ADE) 值的计算非常完善, 所以也被用于 污染物健康风险的计算 ${ }^{[7}$ 。

本文利用CLEA模型，计算了当地居民通过经口 摄入（包括直接食入土壤和食用当地农产品的间接食 入)、皮肤接触以及吸入途径对土壤中PAHs的暴露, 进而对其产生的致癌风险进行了评价, 为评估当地 PAHs的健康风险和CLEA在健康风险评价中的应用 提供数据支持。

\section{2. 材料与方法}

\section{1. 研究区概况与采样}

土壤样品采集地区为苏南地区某镇。该镇工业发达, 主要产业包括生物医药、冶金机械、针织服装等。同 时, 全境内道路四通八达, 随处可见各种集装箱货车、 化工原料货车进而导致当地汽车尾气污染严重。在经 济发展的过程中, 当地企业的废气排放和机动车尾气 排放均会造成多环芳烃在土壤中的累积, 进而对当地 居民的健康产生危害。

本文于 2009 年 9 月在研究区随机布设 29 个样点 进行土壤样品采集。采样时先除去 1-2 cm 枯枝落叶 层, 用土钻采集 $0-10 \mathrm{~cm}$ 的土壤层样品, 将其放入密 封袋中密封运输至实验室, 低温避光保存。运回实验 室后, 将土壤样品平摊在白纸上, 置于阴凉通风处, 温度不高于 $15^{\circ} \mathrm{C}$, 自然风干, 用镊子挑除石块、植 物残体等物质之后进一步碾磨, 样品过 $0.224 \mathrm{~mm}$ ( 70 目）篮, 用于土壤 PAHs 测定。

\section{2. 多环芳烃测定方法及质量控制}

用天平精确称取 $5.0 \mathrm{~g}$ 土壤样品于萃取管中, 并加入 5 种回收率指示物 (荎- $\mathrm{d} 8$ 、屈- $\mathrm{d} 10$ 、葱- $-\mathrm{d} 10$ 、范- $\mathrm{d} 12$ 和 芘-d12) 的混合溶剂, 进行微波提取; 将提取液经 $0.45 \mathrm{~mm}$ 玻璃纤维滤膜真空抽滤分离, 转移到茄形瓶 中, 转换正己烷溶剂, 用真空旋转蒸发仪在 $37^{\circ} \mathrm{C}$ 浓缩至 $1 \mathrm{ml}$; 将浓缩液过硅胶氧化铝层析柱, 用正己 烷淋洗后, 用正己烷和丙酮(体积比 1: 1) 的混合溶 剂淋洗并浓缩至 $1 \mathrm{ml}$, 转换溶剂为正己烷后浓缩至 $1 \mathrm{ml}$, 加入内标物 2 -氟联苯、对三联苯。将浓缩液转
移至气相小瓶中, 用气相色谱质谱联用仪 (GC-MS) 测定 16 种多环芳烃。

实验时每个样品都进行了 3 个平行样品的测定, 样品的回收率在 70\%-120\%之间。16 种 PAHs 标准 曲线的确定系数 $\left(R^{2}\right)$ 在 0.989-0.999 之间。

\section{3. 基于 CLEA 模型的暴露评估}

本文应用 CLEA 软件 1.071 版本进行数据分析。CLEA 模型通过计算目标人群在不同暴露场景下的平均日 暴露量（Average Daily Exposure, ADE）值, 并与健 康标准值 (Health Criteria Values, HCV) 进行比较推 算出土壤标准限值。本文中只利用模型计算不同途径 下的 ADE 值, 计算公式如下:

$$
\begin{aligned}
& \mathrm{ADE}=\frac{\left(I R_{\text {oral }} \times E F_{\text {oral }} \times E D_{\text {oral }}\right)}{B W \times L T}+\frac{\left(I R_{\text {inh }} \times E F_{\text {inh }} \times E D_{\text {inh }}\right)}{B W \times L T} \\
& +\frac{\left(I R_{\text {derm }} \times E F_{\text {derm }} \times E D_{\text {derm }}\right)}{B W \times L T}
\end{aligned}
$$

其中, $\mathrm{ADE}$ 为来源于土壤的某化学物质的人体平均 日摄入量, $\mathrm{mg} /(\mathrm{kg} \bullet \mathrm{d}) ; \mathrm{IR}$ 为化学物质的摄入量, 单位 $\mathrm{mg} / \mathrm{d} ; E F$ 为暴露频率, 单位 $\mathrm{d} / \mathrm{y} ; E D$ 为暴露 时间, 单位为 $y$; $B W$ 为体重, 单位为 $\mathrm{kg}$; $L T$ 为寿 命 75 年。其中, 下标 oral, inh, derm 分别代表经口 摄入、吸入和皮肤接触途径。

\section{4. 模型关键参数及取值}

表 1、2 为模型相关参数取值。当地居住环境为房前 屋后种植农作物, 故设定的暴露场景为生产农产品的 居住用地 (Residence with produce); 土壤质地为粉 黏土 (Slity clay loam); 建筑物类型为接近当地房屋 特征的小排房屋 (Small terraced house); 目标人群为 年龄范围为 0-75 岁的男性和女性。CLEA 模型将年龄 分为 18 个等级: $0-1 、 1-2 、 2-3 、 3-4 、 4-5 、 5-6 、 6-7$ 、 $7-8 、 8-9 、 9-10 、 10-11 、 11-12 、 12-13 、 13-14 、 14-15$ 、 $15-16 、 16-65 、 65-75$ 岁, 其中前 16 个等级为儿童阶 段, 16-65 为成年工作阶段、65-75 岁为退休阶段。 经口摄入途径包括: 土壤、灰尘直接经口摄入、自种 农作物（富集土壤中的污染物）膳食摄取、农作物表 面灰尘摄取。皮肤接触途径包括室内、室外土壤、灰 尘皮肤接触; 吸入途径包括室内、室外灰尘吸入以及 室内、室外土壤挥发气态污染物吸入。表 2 中标注的 相关参数参考了《中国居民营养与健康状况调查报告 
Risk Analysis and Crisis Response in Big Data Era (RAC-16)

之十 2002 营养与健康状况数据集》中一类农村相关 参数或《中国人群暴露参数手册 (成人卷)》中江苏
农村相关参数, 其余均为模型默认值。由于篇幅限制, 部分参数没有按 18 个年龄分级一一列出。

表 1 模型基本参数取值

\begin{tabular}{|c|c|c|c|c|c|c|}
\hline & 用地类型 & 建筑类型 & 土壤类型 & 有机质含量 (\%) & pH & 年龄范围 \\
\hline 取值 & 居住用地（生产农产品） & 小排房屋 & 粉质黏土 & 6 & 7 & $0-75$ \\
\hline
\end{tabular}

表 2 模型高级参数取值

\begin{tabular}{|c|c|c|c|c|c|c|c|c|c|c|}
\hline & \multicolumn{6}{|c|}{ 男 } & \multicolumn{4}{|c|}{ 女 } \\
\hline & $0-1$ & $1-4$ & 4-16 & $16-65$ & $65-75$ & $0-1$ & $1-4$ & $4-16$ & $16-65$ & $65-75$ \\
\hline 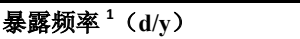 & 180 & 365 & 365 & 365 & 365 & 365 & 365 & 365 & 365 & 365 \\
\hline 暴露频率 ${ }^{2}$ (d/y） & 365 & 365 & 365 & 365 & 365 & 365 & 365 & 365 & 365 & 365 \\
\hline 室内暴露时间（h/d） & 23.0 & 23.0 & 19.0 & \multicolumn{2}{|c|}{$19.1^{[8]}$} & 23.0 & 23.0 & 19.0 & \multicolumn{2}{|c|}{$19.7^{[8]}$} \\
\hline 室外暴露时间（h/d） & 1.0 & 1.0 & 1.0 & \multicolumn{2}{|c|}{$4.7^{[8]}$} & 1.0 & 1.0 & 1.0 & \multicolumn{2}{|c|}{$4.1^{[8]}$} \\
\hline $\begin{array}{l}\text { 土壤-皮肤黏附系数（室内） } \\
\left(\mathrm{mg} /\left(\mathrm{cm}^{2} \cdot \circ \text { day }\right)\right)\end{array}$ & 0.06 & 0.06 & 0.06 & 0.06 & 0.06 & 0.06 & 0.06 & 0.06 & 0.06 & 0.06 \\
\hline $\begin{array}{c}\text { 土壤-皮肤黏附系数（室外） } \\
\left(\mathrm{mg} /\left(\mathrm{cm}^{2} \cdot \circ \text { day }\right)\right)\end{array}$ & 1 & 1 & 1 & 1 & 1 & 1 & 1 & 1 & 1 & 1 \\
\hline 土壤、灰尘摄取率（g/d） & 0.1 & 0.1 & 0.1 & 0.1 & 0.1 & 0.1 & 0.1 & 0.1 & 0.1 & 0.1 \\
\hline 体重（kg） & 6.9 & \multicolumn{2}{|c|}{ 参考文献 [9] } & \multicolumn{2}{|c|}{ 参考文献 [8] } & 5.6 & \multicolumn{2}{|c|}{ 参考文献 [9] } & \multicolumn{2}{|c|}{ 参考文献 ${ }^{[8]}$} \\
\hline 身高（m） & 0.7 & \multicolumn{4}{|c|}{ 参考文献 ${ }^{[8]}$} & 0.7 & \multicolumn{4}{|c|}{ 参考文献 [9] } \\
\hline 呼吸速率 $\left(\mathbf{m}^{3} / \mathbf{d}\right)$ & \multicolumn{3}{|c|}{ 模型默认值 } & 参考文 & 献 ${ }^{[8]}$ & \multicolumn{3}{|c|}{ 模型默认值 } & \multicolumn{2}{|c|}{ 参考文献 ${ }^{[8]}$} \\
\hline $\begin{array}{l}\text { 最大皮肤暴露比率 } \\
\text { (室内) }\left(\mathbf{m}^{2} / \mathbf{m}^{2}\right)\end{array}$ & \multicolumn{10}{|c|}{ 模型默认值 } \\
\hline $\begin{array}{l}\text { 最大皮肤暴露比率 } \\
\text { (室外) }\left(\mathbf{m}^{2} / \mathbf{m}^{2}\right)\end{array}$ & \multicolumn{10}{|c|}{ 模型默认值 } \\
\hline
\end{tabular}

\subsection{6 种 PAHs 的毒性当量计算}

多环芳烃混合物的致癌毒性通常用 $\mathrm{BaP}$ 毒性当量法 来表示, 由公式 (2) 计算得到:

$B E C=\sum_{i=1}^{n} C_{i} \times T E F_{i}$

其中 $\mathrm{C}_{\mathrm{i}}$ 是第 $\mathrm{i}$ 种 PAH 的浓度, $\mathrm{TEF}_{\mathrm{i}}$ 是其对应的 毒性当量因子。TEF 的取值在不同文献中有所不同。 Nisbet 和 LaGoy [10]提出了常见于危害废弃物场地的 17 种 PAHs 的 TEFs。尽管他们因为对部分 PAHs 的 TEFs 赋值缺乏致癌和遗传毒性的证据以及 DahA 的 TEF 值 赋值过高而受到质疑, 但其是现有研究中与美国环境 保护部（USEPA） ${ }^{[11]}$ 的 TEF 最新赋值最为接近的。由 于 USEPA 的研究结果还处于外审阶段, 所以本文采 用了 Nisbet 和 LaGoy 的 TEF 取值。

\subsection{PAHs 致癌风险计算}

人群由于对土壤中 PAHs 的暴露产生的终生致癌风险 (ILCR）可由公式（3）计算: $I L C R=\mathrm{SF} \times \mathrm{ADE}$

(3) 其中, $\mathrm{SF}$ 为 $\mathrm{BaP}$ 的致癌斜率因子 ${ }^{[12]}, 7.3(\mathrm{mg} /(\mathrm{kg} \cdot \mathrm{d}))^{-1}$ 。

\section{3. 结果与讨论}

\section{1. 不同途径 $\mathrm{ADE}$ 值结果}

将 29 个样点的 16 种多环芳烃的 $\mathrm{BaP}$ 当量浓度输 入模型后得到不同途径下的 ADE 结果。表 3、表 4 分 别为女性及男性通过不同途径对土壤中 PAHs 的 ADE 值, 其中 0-16 岁、16-65 岁、65-75 岁分别为少年儿 童阶段 (0-16)、成人阶段 (16-65)、退休阶段 (65-75) 
Risk Analysis and Crisis Response in Big Data Era (RAC-16)

的累积暴露量。三种暴露途径中, 经口摄入的 ADE 值最大, 吸入途径较其它两种途径相比, 基本可以忽 略不计, 这一结果与其它文献 ${ }^{[6]}$ 的结果相似。3 个年 龄阶段的累积 $\mathrm{ADE}$ 值结果排序为: 成人阶段>儿童阶 段〉退休阶段; 但如果考虑年平均 ADE 值, 以女性为 例, 则顺序变为儿童阶段 $\left(1.18 \times 10^{-8}\right)>$ 成人阶段 $\left(7.19 \times 10^{-9}\right)>$ 退休阶段 $\left(7.11 \times 10^{-9}\right)$ 。儿童阶
段的年平均 $\mathrm{ADE}$ 值高于成人, 是因为公式 (1) 里的 BW 体重取值较小, 而其累积 ADE 值小于成人是因为 累积时间（16 年）短导致的。所以，儿童阶段对多 环芳烃的暴露较为敏感。退休阶段的年平均 ADE 值也 低于成人阶段, 这可能是由于其暴露时长 (与土壤接 触的时间) 较低。在相同条件下, 女性的 ADE 值略高 于男性, 可能是女性的体重低于男性导致的。

表 3 女性居民通过不同途径对土壤中 PAHs 的 ADE 值（单位 $\mathrm{mg} /(\mathrm{kg} \cdot \mathrm{d})$ )

\begin{tabular}{|c|c|c|c|c|c|}
\hline & \multicolumn{2}{|c|}{ 经口摄入 } & \multirow{2}{*}{$\begin{array}{c}\text { 皮肤接触 } \\
\text { (Dermal） } \\
\left(10^{-9}\right)\end{array}$} & \multirow{2}{*}{$\begin{array}{c}\text { 吸入 } \\
\text { (Inhalation) } \\
\left(10^{-11}\right)\end{array}$} & \multirow{2}{*}{$\begin{array}{l}\text { 总 } \\
\left(10^{-8}\right)\end{array}$} \\
\hline & $\begin{array}{c}\text { 经口直接摄入 } \\
\left(10^{-7}\right)\end{array}$ & $\begin{array}{c}\text { 膳食摄入 } \\
\left(10^{-9}\right)\end{array}$ & & & \\
\hline \multirow{2}{*}{ 0-16 } & 1.06 & 11.5 & 71.0 & 31.5 & 18.8 \\
\hline & $0.32-2.36$ & $3.45-25.6$ & $21.3-158$ & $9.46-70.3$ & $5.66-42.0$ \\
\hline \multirow{2}{*}{$16-65$} & 1.12 & 21.4 & 218 & 33.9 & 35.2 \\
\hline & $0.34-2.51$ & $6.44-47.8$ & $59.9-487$ & $10.2-75.6$ & $10.6-78.6$ \\
\hline \multirow{2}{*}{$65-75$} & 0.23 & 4.38 & 43.7 & 6.92 & 7.11 \\
\hline & $0.069-0.51$ & $1.31-9.76$ & $65.5-97.4$ & $2.08-15.4$ & $2.13-15.9$ \\
\hline \multirow{2}{*}{$16-75$} & 1.35 & 25.8 & 262 & 40.8 & 42.4 \\
\hline & $0.41-3.02$ & $7.75-57.6$ & $13.1-584$ & $12.3-91.0$ & $12.7-94.4$ \\
\hline \multirow{2}{*}{$0-75$} & 2.41 & 37.3 & 333 & 72.3 & 61.2 \\
\hline & $0.72-5.38$ & $11.2-83.2$ & 78.6-742 & $21.7-161$ & $18.4-136$ \\
\hline
\end{tabular}

表 4 男性居民通过不同途径对土壤中 PAHs 的 ADE 值 (单位 $\mathrm{mg} /(\mathrm{kg} \cdot \mathrm{d}$ ) )

\begin{tabular}{|c|c|c|c|c|c|}
\hline 途径 & \multicolumn{2}{|c|}{ 经口摄入 } & \multirow{2}{*}{$\begin{array}{c}\text { 皮肤接触 } \\
\text { (Dermal） } \\
\left(10^{-9}\right)\end{array}$} & \multirow{2}{*}{$\begin{array}{c}\text { 吸入 } \\
\text { (Inhalation) } \\
\left(10^{-11}\right)\end{array}$} & \multirow{2}{*}{$\begin{array}{c}\text { 总 } \\
\left(10^{-8}\right)\end{array}$} \\
\hline 年龄 & $\begin{array}{c}\text { 经口直接摄入 } \\
\left(10^{-7}\right)\end{array}$ & $\begin{array}{c}\text { 膳食摄入 } \\
\left(10^{-9}\right)\end{array}$ & & & \\
\hline \multirow{2}{*}{$0-16$} & 1.01 & 11.5 & 69.8 & 31.5 & 18.2 \\
\hline & $0.30-2.24$ & $3.45-25.6$ & $20.9-156$ & $9.47-70.3$ & $5.47-40.6$ \\
\hline \multirow{2}{*}{$16-65$} & 0.97 & 21.4 & 208 & 34.7 & 32.7 \\
\hline & $0.29-2.16$ & $6.44-47.8$ & $62.5-464$ & $10.4-77.4$ & $9.81-72.9$ \\
\hline \multirow{2}{*}{$65-75$} & 0.20 & 4.38 & 42.1 & 7.08 & 6.63 \\
\hline & $0.059-0.44$ & $1.31-9.76$ & $12.6-93.8$ & $2.31-15.8$ & $1.99-14.8$ \\
\hline \multirow{2}{*}{$16-75$} & 1.17 & 25.8 & 250 & 41.8 & 39.3 \\
\hline & $0.35-2.60$ & $7.75-57.6$ & $75.1-558$ & $12.5-93.2$ & $11.8-87.7$ \\
\hline \multirow{2}{*}{$0-75$} & 2.17 & 37.3 & 320 & 73.3 & 57.5 \\
\hline & $0.65-4.87$ & $11.2-83.2$ & $96.1-714$ & $22.0-164$ & $17.3-128$ \\
\hline
\end{tabular}

\section{2. CLEA 健康风险评价}

根据公式 (3), 由人体对土壤中 PAHs 的暴露量 $\mathrm{ADE}$ 值计算得到相应的致癌风险。总的来说, 不同性 别的所有年龄阶段的健康风险均超出了可接受的致
癌风险水平 $\left(10^{-6}\right)$, 应当引起重视。不同途径的健康 风险 (图 1) 与 $\mathrm{ADE}$ 值的规律一致。经口摄入是土壤 中多环芳烃对人类健康产生危害的最主要途径; 人的 一生中风险最高的年龄阶段是成人阶段, 因为成人阶 段的暴露时段最长; 儿童是多环芳烃的敏感人群; 女 性的健康风险高于男性。 
Risk Analysis and Crisis Response in Big Data Era (RAC-16)

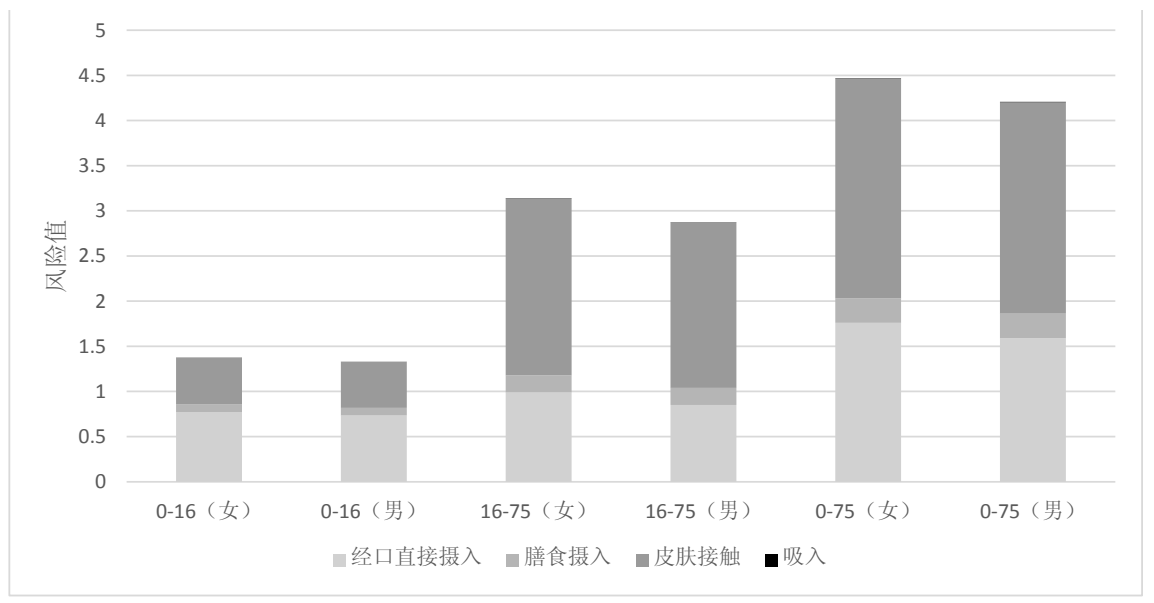

图 1 不同途径人体对多环芳烃暴露的健康风险 $\left(10^{-6}\right)$

\section{3. 不确定性分析}

在应用 CLEA 模型时, 由于缺少研究区相关数据无法 将一些参数本地化, 比如土壤性质参数 $(\mathrm{pH}$ 、有机质 含量)、建筑物具体参数、男性及女性不同年龄阶段 的部分暴露参数、多环芳烃的富集转运系数等。这些 会给风险分析带来一定的不确定性。另外模型无法对 多个样本点的土壤浓度数据进行统计分析, 仅用算数 均值代表研究区的平均污染水平, 也会损失很多有用 信息。

\section{4. 结论}

本文利用 CLEA 模型对土壤中 PAHs 对人体的健康风险 进行分析, 结果表明, 经口摄入和皮肤接触是危害人 体健康的主要途径。该地区人群的健康风险值超过推 荐的致癌风险水平 $\left(10^{-6}\right)$, 应当引起关注。其中, 16-75 岁人群的致癌风险最高、女性致癌风险略高于男性。

\section{Acknowledgements}

This work was supported by the Key Technologies R\&D Program for the 12th Five-Year Plan (No.
2012BAJ24B04) of the Ministry of Science and Technology of People's Republic of China.

\section{致谢}

本研究得到了国家科技部 “十二五” 科技支撑课题 (No. 2012BAJ24B04)的资助。

\section{参考文献}

[1] 张生银. 李双林.董贺平 等.南黄海中部表层沉 积物中多环芳烃分布特征及来源分析.中国环境 科学.1263-1270.2013.

[2] USEPA. Appendix A to 40 CFR. Part 423- 126 Priority Pollutants. 2003.

[3] M. Mari, J. Díaz-Ferrero, M. Schuhmacher, M. Nadal, J. L. Domingo. Health risks of environmental exposure to $\mathrm{PCDD} / \mathrm{Fs}$ near a hazardous waste incinerator in catalonia, spain. Journal of Risk Analysis and Crisis Response. 2013, 3(2):77-87.

[4] 姜林. 钟茂生. 张丹等. 污染场地土壤多环芳烃 (PAHs)生物可利用浓度的健康风险评价方法, 生态环境学报.1168-1175.2011

[5] Environment Agency. Updated technical background to the CLEA model. 2009

[6] 夏风英. 曹云者. 李政一等. 用 RBCA 和 CLEA 模型推导土壤中苯并 [a]萠的标准值. 环境科学 
Risk Analysis and Crisis Response in Big Data Era (RAC-16)

研究. 1445-1451.2009.

[7] 贾晓洋。姜林.夏天翔等. RBCA、CLEA 及 CalTOX 模型在苯并 [a]萠污染场地健康风险评 估中的应用比较.生态毒理学报. 277-284.2012.

[8] 环境保护部.中国人群暴露参数手册（成人卷）, 中国环境出版社.北京. 2013 .

[9] 金水高.中国居民营养与健康状况调查报告之十 2002 营养与健康状况数据集. 人民卫生出版社. 北京. 2008 .

[10] I.C.T. Nisbet. P.K. LaGoy. Toxic equivalency factors (TEFs) for polycyclic aromatic hydrocarbons (PAHs). Regul Toxicol Pharm, 1992, 290-300.

[11] California EPA (California Environmental Protection Agency). No Significant Risk Levels (NSRLs) for the Proposition 65 carcinogens benzofluoranthene, benzo[j]fluoranthene, chrysene, dibenzo[a,h]pyrene, dibenzo[a,i]pyrene, and 5 -methylchrysene by the oral route. Office of Environmental Health Hazard Assessment, Reproductive and Cancer Hazard Assessment Section. Oakland. CA. 2004.

[12] USEPA. IRIS Toxicological Review of Benzo[a]pyrene (External Review Draft). 2014. 